\title{
Comparison between optimized topologies of permanent magnet thrust bearings with back-iron
}

\author{
Maxence VAN BENEDEN*, Virginie KLUYSKENS* and Bruno DEHEZ* \\ * Center for Research in Mechatronics (CEREM) \\ Universit catholique de Louvain (UCL), Louvain-la-Neuve, Belgium \\ E-mail: maxence.vanbeneden@uclouvain.be
}

Received: 27 January 2017; Accepted: 26 April 2017

\begin{abstract}
This paper deals with the optimization and the comparison of passive magnetic thrust bearings made up of radial stacks of permanent magnet rings. Various topologies are considered, depending on the polarization direction of the permanent magnet rings and the presence of back-iron. The coulombian approach and the method of image charges are used to determine the load capacity of topologies with back-iron. On this basis each topology is optimized in order to minimize the permanent magnet volume for a fixed load capacity, airgap thickness and remanent flux density. Varying these parameters, scaling laws of the optimum permanent magnet ring dimensions are derived to allow fast sizing and comparison of the topologies. The latter highlights that the topology with Halbach configuration and back-iron is the most performant, but that the topology with axial polarization is almost as good.
\end{abstract}

Keywords : Bearings, Back-iron, Coulombian approach, Method of image charges, Optimization, Permanent magnets

\section{Introduction}

Passive thrust bearings are made of permanent magnet (PM) rings. The PM rings can be radially or axially stacked (Yonnet, 1991) in order to increase the axial force. Topologies with (Sotelo, de Andrade and Ferreira, 2007) or without (Yoo, Kim, Kim, Lee, Bae, and Noh, 2011) soft magnetic materials are studied in the literature. The use of highly permeable material (back-iron) increases the performance of PM bearings for a same PM volume (Jungmayr, Marth, Amrhein, Berroth and Jeske, 2014).

From another point of view, the actual trends consists in limiting the use of PM material, because of the limited amount of resource for rare earth material and because of economical reasons. It is thus important when working with PM thrust bearings to take care of minimizing the required volume of PM.

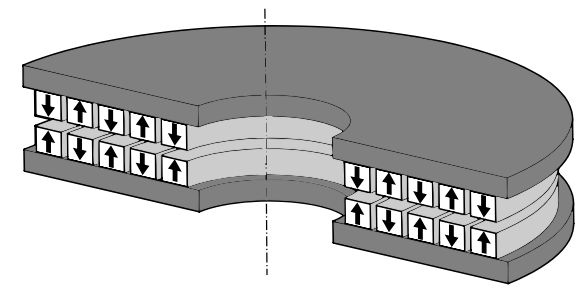

Fig. 1 Thrust bearing with radially stacked permanent magnet rings, and with back-iron (in dark grey).

Different models based on coulombian approach (Yonnet, 1978), (Ravaud, Lemarquand, and Lemarquand, 2009) are developed to predict the forces between magnets. In the article of Jungmayr, Marth, Amrhein, Berroth and Jeske (2014), 
the method of image charges is used to model the influence of the soft material.

Parametric optimizations of particular PM thrust bearings can be found in the literature (Moser and Bleuler, 2006) (Bekinal, Anil, and Jana, 2013) (Yoo, Kim, Kim, Lee, Bae, and Noh, 2011), but to the author's best knowledge there is no complete and transverse study comparing the performances between the different topologies.

This paper presents passive thrust bearings made of PM rings radially stacked like shown in Fig. 1. The influence of back-iron is studied. Different topologies are considered with axial and/or radial polarization. Each one of these topologies is optimized with the objective to minimize the required volume of PM needed to carry a given axial load. Their performances are compared between them and with the performances of ironless PM thrust bearing (Van Beneden, Kluyskens and Dehez, 2015 and 2017). This paper presents also scaling laws that allows fast sizing of the optimal magnet dimensions.

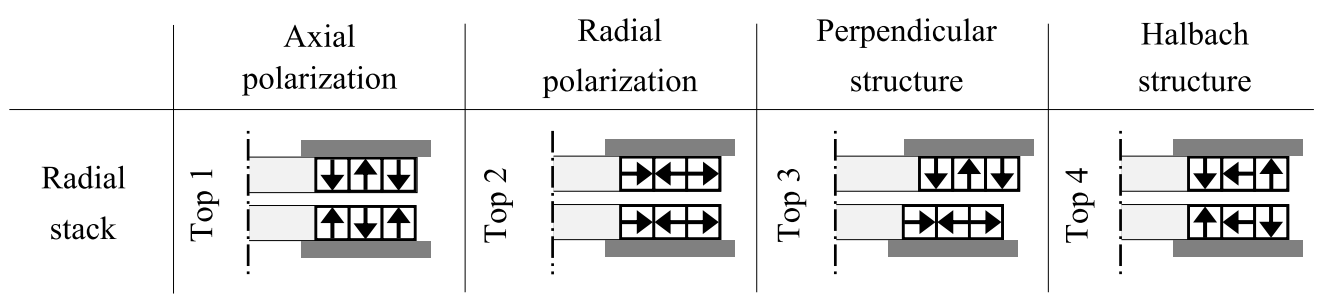

Fig. 2 The different considered topologies, with back-iron (in dark grey).

The structure of this paper will be as follows. First, the topologies of PM thrust bearings considered in this paper are presented in section 2 . Then in section 3, these topologies are modelled and this model is validated by finite element model. In section 4, each topology is optimized separately with the objective to minimize the magnet volume. The influence of the number of pairs of rings, the load, the air gap and the remanent flux densities is shown. Finally, topologies with and without back-iron are compared in section 5 .

\section{Bearing topologies}

The studied PM bearings are made of two radial stacks of PM rings, disposed coaxially, one of the stacks being linked to the rotor, and the other one to the stator. The polarization of each magnet ring can be axial or radial. Combinations of these polarizations are also investigated, like the perpendicular structure or the Halbach structure (Halbach,1980) . For each case, an iron frame is placed at the back of the structure. The four different considered topologies are illustrated in Fig. 2.
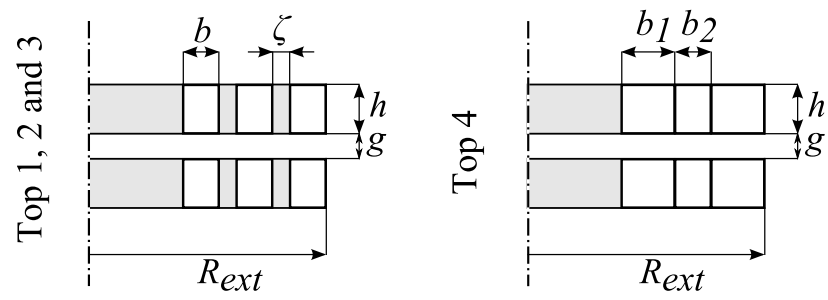

Fig. 3 The parameters of the optimization : $h$ the height of the ring section $; b$ th width of the ring section $\zeta \zeta$ the gap between rings $; g$ the air gap $; R_{\text {ext }}$ the external radius.

The dimensions of each topology can be described by 4 parameters illustrated in Fig. 3 . They are the height $h$ and the width $b$ of the ring section, the gap $\zeta$ in between the rings and the external radius $R_{\text {ext }}$. The gap $\zeta$ is considered because it improves the axial force (Yoo, Kim, Kim, Lee, Bae, and Noh, 2011). In the case of the Halbach structure (Top 4), there is no space between rings but the width of the ring section can be different for both polarization direction : $b_{1}$ and $b_{2}$. The last parameter is the total length $L$ of PM illustrated in Fig. 4. Different magnet bearings are presented with the same total length but different numbers of pairs of rings $N$. This parameters depends on the others and is calculated by :

$$
L=2 \times \pi \times\left(\sum_{i=1}^{N} R_{s, i}+\sum_{i=1}^{N} R_{r, i}\right),
$$


where $R_{s, i}$ and $R_{r, i}$ are the mean radius of PM rings from 1 to $N$ of the stator and the rotor respectively. This parameter is presented because of its interest in section 4.

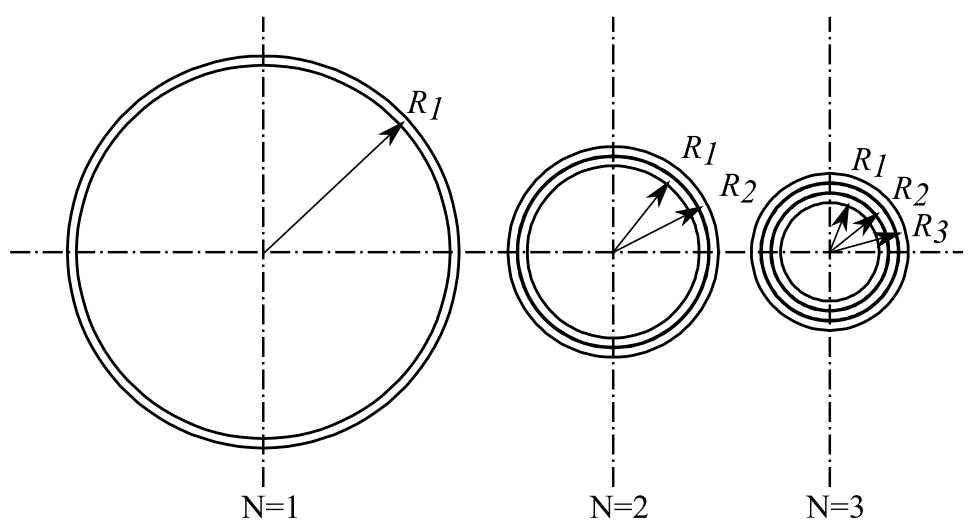

Fig. 4 Top view of of PM bearing with the same total length $L$ but different number of rings $N$.

\section{Modelling}

In the literature, we can find different models based on coulombian approach to evaluate the axial force between magnets for the three polarization possibilities (axial, radial and perpendicular). A 2D plane approach was developed by Yonnet (Yonnet, 1978). The complete resolution is analytical but the curvature effect is not considered. As shown in a previous article (Van Beneden, Kluyskens and Dehez, 2015 and 2017), the curvature effect can be neglected by considering topologies with $R_{e}>2 b+g$ where $R_{e}$ is the mean radius of the air gap.

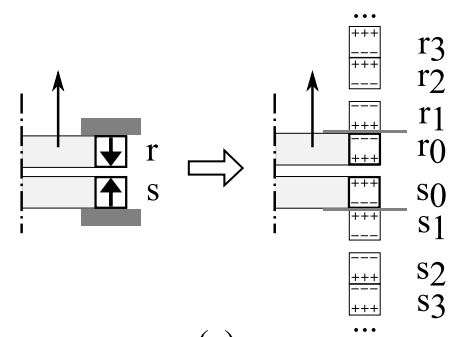

(a)

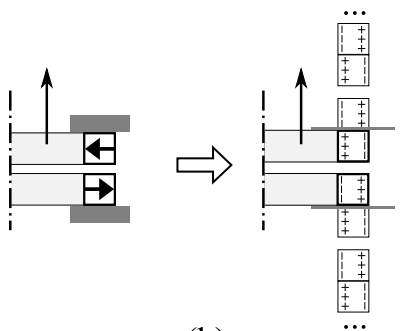

(b)

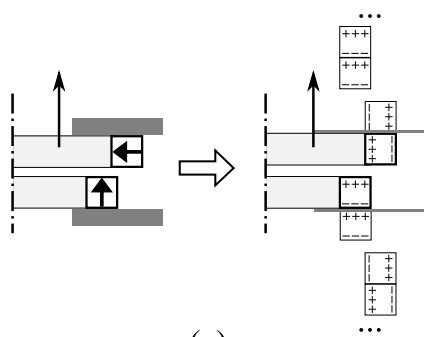

(c)

Fig. 5 Illustration of the Coulombian approach and method of image charges for (a) axial polarization, (b) radial polarization and (c) perpendicular polarization.

The back-iron can be modelled by the method of image charges (Plonsey, 1961) (Jungmayr, Marth, Amrhein, Berroth and Jeske, 2014) as shown in Fig. 5, considering infinite the magnetic permeability of the back-iron. On this basis, the axial force $f_{r, s}$ exerted on the ring $r$ by the ring $s$ is calculated by the double sum of the forces exerted on the rotor rings $r_{i}$ by the stator rings $s_{j}$ (real for $i, j=0$ and virtual $i, j \geq 1$ ):

$$
f_{r, s}=\sum_{i=0}^{N_{\text {mirror }}=\infty} \sum_{j=0}^{N_{\text {mirror }}=\infty} f_{r_{i}, s_{j}},
$$

The convergence of the sum is studied by comparing the results for different values of $N_{\text {mirror }}$. The convergence of this sum is shown in Fig. 6 . The axial force calculation converges for $N_{\text {mirror }} \geq 3$. This convergence depends on the shape of the ring section : if the height $h$ is small in comparison of the air gap $g$ and the width $b$, the convergence appears for a bigger $N_{\text {mirror }}$. This will be verified a posteriori.

The total axial force $F_{z}$ of the topologies made of stacks of $N$ PM rings of Fig. 2 is calculated by the double sum 


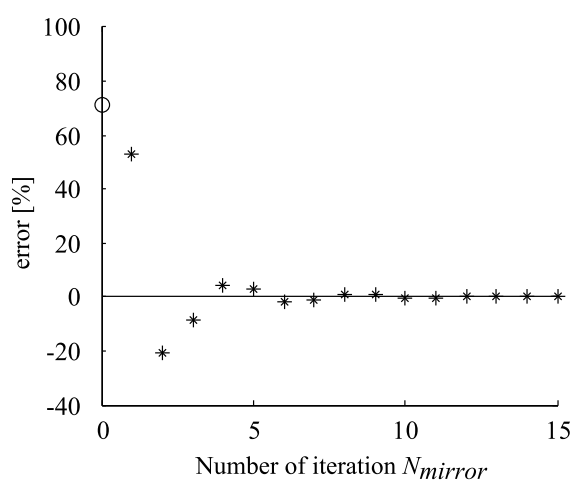

Fig. 6 Convergence of the axial force $F_{z}$ with the number on partial sum $N_{\text {mirror }}$ in $\%$ of the relative error :

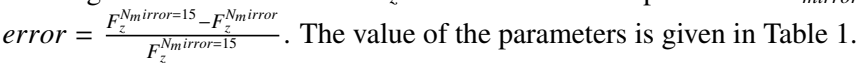

Table 1 The value of the parameters for Fig. 6 .

\begin{tabular}{ccc} 
& Parameter & Value \\
\hline$h$ & Magnet section height & $7.5 \mathrm{~mm}$ \\
$b$ & Magnet section width & $15 \mathrm{~mm}$ \\
$R_{e x t}$ & External radius & $400 \mathrm{~mm}$ \\
$B_{r}$ & Remanent flux density & $1 \mathrm{~T}$ \\
$g$ & Air gap & $2 \mathrm{~mm}$ \\
$N$ & Number of rings & 1 \\
\hline
\end{tabular}

from 1 to $N$ of the force $f_{r, s}$ exerted by the stator rings $s$ on the rotor rings $r$ :

$$
F_{z}=\sum_{s=1}^{N} \sum_{r=1}^{N} f_{r, s} .
$$

As shown in Fig. 7, the model, in which $N_{\text {mirror }}$ is fixed to 3 , is validated by finite element method (FEM), in which the iron permeability is set to 5000 .

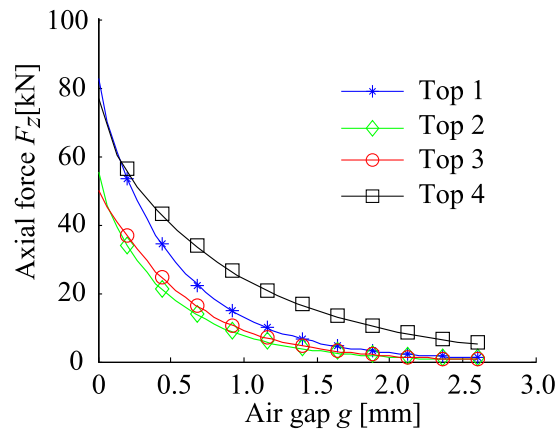

Fig. 7 Axial force $F_{z}$ for each topology as a function of the air gap $g$. Points : FEM ; Lines : analytical model. The value of the parameters is given in Table 2 .

\section{Optimization}

The optimization of the different topologies is made by a hybrid algorithm that combines a NSGAII and a simplex with penalty method algorithms. The objective function is to minimize the PM volume $V$. The constraint is to carry the axial load $F_{z} \geq F_{z, \text { ref }}$. The optimization parameters are the magnet section height $h$, the magnet section width $b$ for Top 1 , Top 2 and Top 3 and $b_{1}$ and $b_{2}$ for Top 4, the magnet section space $\zeta$ and the external radius $R_{e x t}$. The fixed parameters are the air gap $g$, the remanent flux density $B_{r}$ and the number of rings by stack $N$. 
Table 2 The value of the parameters of Fig. 7.

\begin{tabular}{ccc} 
& Parameter & Value \\
\hline$h$ & Magnet section height & $12 \mathrm{~mm}$ \\
$b$ & Magnet section width & $15 \mathrm{~mm}$ \\
$\zeta$ & Magnet section space & $2 \mathrm{~mm}$ \\
$R_{\text {ext }}$ & External radius & $400 \mathrm{~mm}$ \\
$B_{r}$ & Remanent flux density & $1 \mathrm{~T}$ \\
$N$ & Number of rings & 7 \\
\hline
\end{tabular}

In a first step, the influence on the optimal parameters of the number of rings $N$ is studied. Secondly, the influence of the other input parameters $\left(F_{z, r e f}, g\right.$ and $\left.B_{r}\right)$ on the optimal parameters are studied. From these results, scaling laws on these parameters are derived.

\subsection{Influence of the number of rings}

The influence of the number of rings $N$ is studied in this section. Each topology is optimized separately. The number of PM rings $\mathrm{N}$ is increased from $3,5, \ldots$ to 29 . The other input parameters are set to $F_{z, r e f}=50[\mathrm{kN}], g=1[\mathrm{~mm}]$ and $B_{r}=1[T]$.

As shown in Fig. 8, the optimal magnet volume $V_{o p t}^{N}$ converges asymptotically with the number of pairs of rings $N$. This convergence is due to a decrease of the edge effect with the number of pairs of rings $N$. If the edge effects are neglected, there is a theoretical optimal value of the minimum magnet volume $V_{\text {opt }}$. When the number of rings $N$ continues to increase, the volume converges towards this theoretical optimal volume $V_{\text {opt }}$ until the activation of other constraints like geometrics constraints on the minimum radius leading to an increase of the minimum magnet volume again.

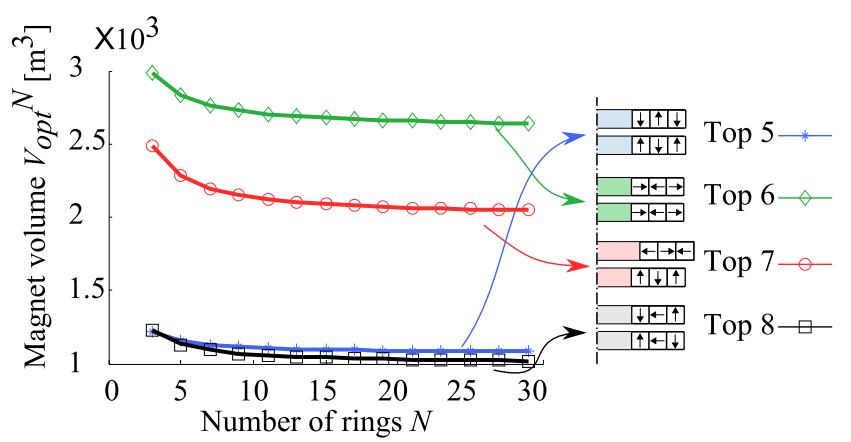

Fig. 8 Evolution of the minimum magnet volume $V_{o p t}^{N}$ with the number of pairs of rings $N$.

Figure 9 shows the evolution of the optimal geometrical parameters with the number of pairs of rings $N: h_{o p t}^{N}, b_{o p t}^{N}$, $\zeta_{o p t}^{N}, L_{o p t}^{N}$. These parameters converge also with the number of pairs of rings $N$ until a theoretical optimal value $: h_{o p t}$, $b_{\text {opt }}, \zeta_{\text {opt }}, L_{\text {opt }}$. The use of the total length $L$ as parameters is interesting because it converges with the number of rings in contrary of the radius.

In order to take into account the influence of the number of pairs of rings $N$ on the optimal value of the parameters, correction functions can be used, such that:

$$
h_{o p t}^{N}=h_{o p t} f_{h}(N) \quad b_{o p t}^{N}=b_{o p t} f_{b}(N) \quad \zeta_{o p t}^{N}=\zeta_{o p t} f_{\zeta}(N) \quad L_{o p t}^{N}=L_{o p t} f_{L}(N),
$$

where $h_{o p t}^{N}, b_{o p t}^{N}, \zeta_{o p t}^{N}$ and $L_{o p t}^{N}$ are the optimal magnet dimensions for a given number of rings $N$ and $f_{h}(N), f_{b}(N), f_{\zeta}(N)$ and $f_{L}(N)$ are the corrections functions to take into account the influence of the number of rings $N$ for $N=[3 ; \infty[$ :

$$
f_{i}(N)=1+\lambda_{i} \frac{1}{N}+\mu_{i} \frac{1}{N^{2}},
$$



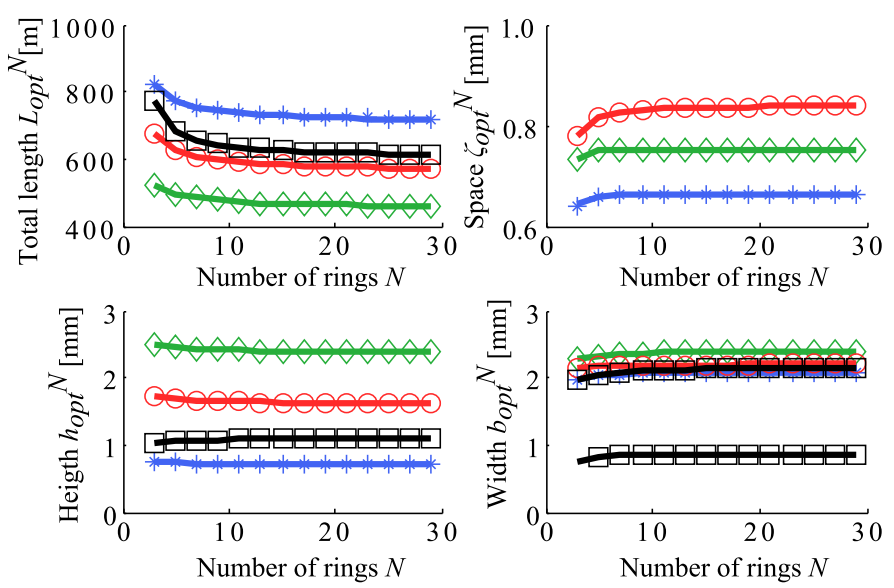

Fig. 9 Evolution of the geometrical parameters : the height $h_{o p t}^{N}$ and the width $b_{o p t}^{N}$ of the ring section, the space between the ring $\zeta_{o p t}^{N}$ and the total length $L_{o p t}^{N}$ with the number of rings $N$.

with $i=h, b, \zeta$ or $L$.

These correction functions $f_{i}(N)$ are non physics based and are chosen to fit with the results of Fig. 9. Nevertheless, they converge to 1 for increasing values of $N$. The value of the coefficients $\lambda_{i}$ and $\mu_{i}$ is calculated by the root mean squared error (RMSE) method. The RMSE is calculated by taking into account the error on the parameters but also on the volume :

$$
R M S E=\sqrt{\sum\left(10 \epsilon_{V}^{2}+\epsilon_{h}^{2}+\epsilon_{b}^{2}+\epsilon_{L}^{2}\right)}
$$

where $\epsilon_{V}, \epsilon_{h}, \epsilon_{b}$ and $\epsilon_{L}$ are the relative error of the volume, the section height, the section width and the total length respectively. The weight of 10 on the volume is set because of its importance to guarantee the axial force $F_{z, \text { ref }}$. For the section space $\zeta$, the RMSE is calculated independently.

The optimal results are shown in Table 3 . The value of the constants $\lambda_{i}$ and $\mu_{i}$ and the RMSE are shown in Table 4.

Table 3 Optimal result for each topology

\begin{tabular}{c|c|c|c|c} 
& Top 1 & Top 2 & Top 3 & Top 4 \\
\hline$V_{\text {opt }}\left[\mathrm{dm}^{3}\right]$ & 1.07 & 2.60 & 2.00 & 1.00 \\
\hline$h_{\text {opt }}[\mathrm{mm}]$ & 0.72 & 2.38 & 1.62 & 1.09 \\
\hline$b_{\text {opt }}[\mathrm{mm}]$ & 2.10 & 2.41 & 2.21 & $b_{1}: 2.19 ; b_{2}: 0.84$ \\
\hline$\zeta_{\text {opt }}[\mathrm{mm}]$ & 0.66 & 0.75 & 0.84 & 0 \\
\hline$L_{\text {opt }}[\mathrm{m}]$ & 707 & 454 & 562 & 605
\end{tabular}

Table 4 Coefficients of the correction functions $f_{i}(N)$

\begin{tabular}{c|c|c|c|c} 
& Top 1 & Top 2 & Top 3 & Top 4 \\
\hline$\lambda_{h}$ & 0.0883 & 0.1078 & 0.1924 & -0.0478 \\
$\mu_{h}$ & 0.0730 & 0.0906 & 0.0214 & -0.4499 \\
\hline$\lambda_{b}$ & -0.1462 & -0.1470 & -0.0672 & $b_{1}:-0.3914 ; b_{2}: 0.3619$ \\
$\mu_{b}$ & -0.0314 & -0.0289 & -0.0646 & $b_{1}: 0.2904 ; b_{2}:-1.8926$ \\
\hline$\lambda_{\zeta}$ & 0.0945 & 0.1579 & -0.0190 & 0 \\
$\mu_{\zeta}$ & -0.5317 & -0.6631 & -0.5867 & 0 \\
\hline$\lambda_{L}$ & 0.4571 & 0.4617 & 0.5063 & 0.4323 \\
$\mu_{L}$ & 0.0987 & 0.0337 & 0.2685 & 1.1709 \\
\hline $\mathrm{RMSE}$ & $0.13 \%$ & $0.22 \%$ & $0.29 \%$ & $1.03 \%$
\end{tabular}

The link between the volume of PM and the geometrical parameters are given in:

$$
\begin{aligned}
& V=L \times h \times b \text { for Top }=1,2 \text { and } 3 \\
& V=L \times h \times \frac{b_{1}+b_{2}}{2} \times\left(1+\frac{1}{2} \frac{b_{1}-b_{2}}{b_{1}+b_{2}}\left(\frac{1-(-1)^{N}}{N}-\frac{4 \pi N}{L} \frac{b_{1}+b_{2}}{4}\left(1+(-1)^{N}\right)\right)\right) \quad \text { for Top }=4
\end{aligned}
$$




\subsection{Influence of the load, the air gap and the remanent flux density}

The influence of the load $F_{z, r e f}$, the air gap $g$ and the remanent flux density $B_{r}$ are studied. Each topology is optimized separately. The number of pairs of rings is set to $N=11$ but the optimal values obtained from the optimization process are corrected through the correction functions introduced in the previous section. The range of variation of the

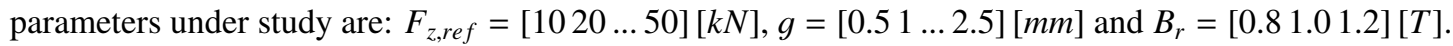

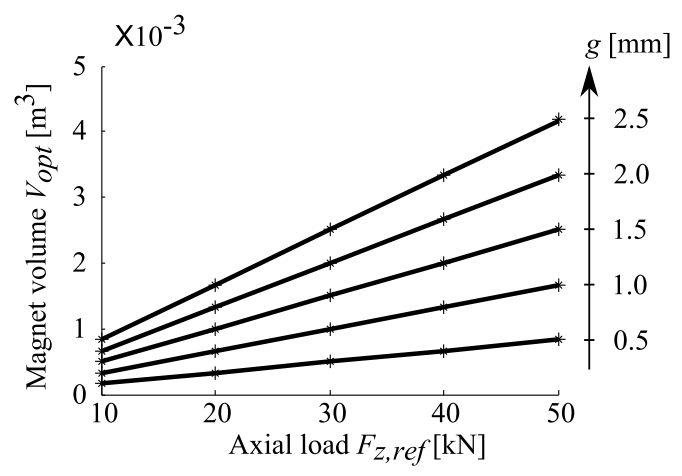

Fig. 10 Evolution of the optimal volume with the load $F_{z, \text { ref }}=[1020 \ldots 50][\mathrm{kN}]$ and the air gap $g=\left[\begin{array}{llll}0.5 & 1.0 & \ldots & 2.5\end{array}\right][\mathrm{mm}]$.

The result for Top 1 is given in Fig. 10 and Fig. 11. From these results, scaling laws of the optimal magnet volume and magnet parameters can be derived as a function of the load, the air gap and the remanent flux density. The scaling laws for the magnet volume and magnet parameters are given in Eq. (9) and Eq. (10) respectively. The same procedure is presented for topologies without back-iron in (Van Beneden, Kluyskens and Dehez, 2017).
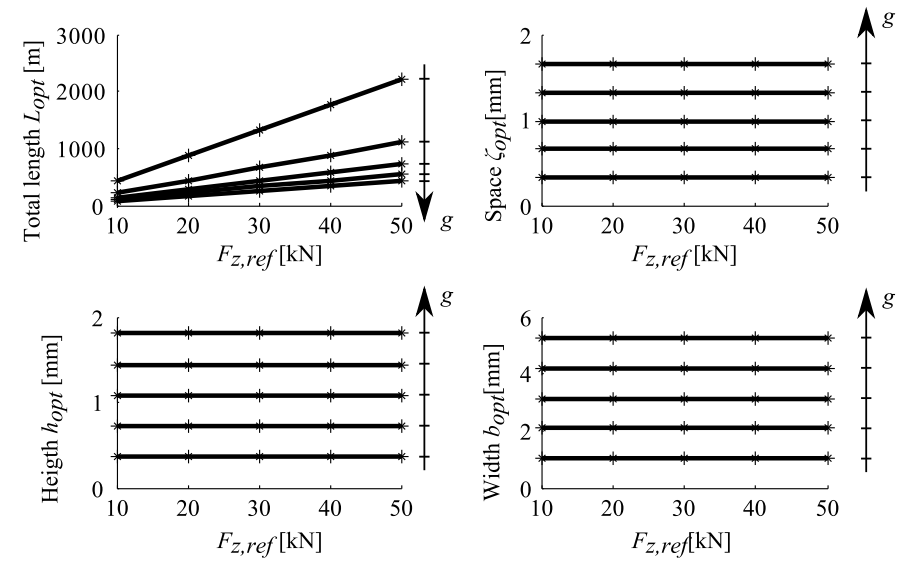

Fig. 11 Evolution of the geometrical parameters with the load $F_{z, \text { ref }}=$ $[1020 \ldots 50][\mathrm{kN}]$ and the air gap $g=[0.51 .0 \ldots 2.5][\mathrm{mm}]$.

$$
\begin{aligned}
& V_{o p t}=\alpha \times g \times F_{z, r e f} \times \frac{1}{B_{r}^{2}} . \\
& h_{o p t}=\beta \times g \quad b_{o p t}=\gamma \times g \quad \zeta_{o p t}=\delta \times g \quad L_{o p t}=\epsilon \times \frac{F_{z, r e f}}{g} \times \frac{1}{B_{r}^{2}} .
\end{aligned}
$$

with $\alpha, \beta, \gamma, \delta$ and $\gamma$ the scaling factors. The latter depend on the bearing topology and they are given in Table 5 .

Table 5 Scaling factors for the optimum PM volume and optimum parameters.

\begin{tabular}{c|c|c|c|c|c} 
& $\alpha \times 10^{-5}\left[\frac{m^{2} T^{2}}{N}\right]$ & $\beta[-]$ & $\gamma[-]$ & $\delta[-]$ & $\epsilon \times 10^{-5}\left[\frac{m^{2} T^{2}}{N}\right]$ \\
\hline Top 1 & 2.13 & 0.721 & 2.09 & 0.660 & 1.41 \\
Top 2 & 5.20 & 2.39 & 2.40 & 0.746 & 0.907 \\
Top 3 & 4.01 & 1.62 & 2.21 & 0.839 & 1.13 \\
Top 4 & 2.08 & 1.08 & $2.17 ; 0.830$ & 0 & 1.21
\end{tabular}


Notice that for the Halbach structure (Top 4), there is two scaling factors $\gamma: 2.17$ linked to the axially polarized PM rings and 0.844 linked to the radially polarized PM rings.

\section{Comparison}

In this section, topologies with and without back-iron are compared based on the minimum magnet volume. For this purpose, the optimal magnet volumes are expressed in percentage of the optimal magnet volume of the Halbach topology with back-iron (Top 4BI) :

$$
\frac{V_{o p t}^{T o p i}}{V_{o p t}^{T o p} 4 B I}=\frac{\alpha^{T o p i} \times g \times F_{z, r e f} \times \frac{1}{B_{r}^{2}}}{\alpha^{T o p 4 B I} \times g \times F_{z, r e f} \times \frac{1}{B_{r}^{2}}}=\frac{\alpha^{T o p i}}{\alpha^{T o p 4 B I}},
$$

with $i$ the different topologies : without back-iron 1, 2, 3, 4 and with back-iron 1BI, 2BI, 3BI and 4BI. As shown in Eq. (11), the ratio between the optimal volume are independent of the load, the air gap and the remanent flux densities. The results for topologies without back-iron are taken from (Van Beneden, Kluyskens and Dehez, 2017). The results of the comparison are summarized in Table 6 where $\Delta$ is the gain in percentage of PM volume using back-iron, for each topology.

Table 6 Comparison between topologies with and without back-iron in percentage of the Halbach topology with back-iron $V_{o p t}^{T o p} 4 B I . \Delta$ is the difference between topology with BI and without BI.

\begin{tabular}{c|c|c|c}
$\frac{V_{\text {opt }}^{\text {Top i }}}{V_{\text {op }}^{\text {Top }} \text { 4BI }}$ & Without BI & With BI & $\Delta$ \\
\hline Top 1 & 174 & 103 & 71 \\
Top 2 & 174 & 250 & -76 \\
Top 3 & 175 & 193 & -17 \\
Top 4 & 101 & 100 & 1
\end{tabular}

On the one hand, we can see that the use of back-iron is advantageous for some topologies (Top 1 and Top 4) but is harmful for others (Top 2 and 3). For Top 2 and Top 3, with radial polarization and perpendicular polarization respectively, the back-iron deviates the magnetic flux produced by PM out of the air gap. Therefore, the axial force is impacted negatively. This effect is stronger for Top 2. For Top 1 and Top 4, with axial polarization and Halbach structure respectively, the effect of the back-iron is positive since it closes the magnetic circuit followed by the magnetic flux behind the PM. This effect is stronger for Top 1 .

On the other hand, we can see that Halbach structure with back-iron is the best (Top 4BI). The use of back-iron is slightly benefit (1\%) for this topology. By contrast, the use of back-iron for topology with axial polarization (Top 1) is large (71\%). Consequently the minimum PM volume required in Top 1BI, with axially magnetized permanent rings, and back-iron, is identical to the needed PM volume in Top 4, for a Halbach structure without back-iron. This volume is even very close to the needed volume for Top 4BI, the Halbach structure with back-iron. Considering that Halbach structure is often difficult to produce, Top 1BI is a competitive topology.

\section{Conclusion}

This paper compares different PM thrust bearing topologies. In a first step, the different topologies with back-iron are presented. The Coulombian approach and method of image charges are used and validated by finite element method. Secondly, the influence of the number of rings $N$ on the optimum for each topology is shown and can be neglected for $N$ large enough. Thirdly, the influence of the load $F_{z, r e f}$, the air gap $g$ and the remanent flux $B_{r}$ is evaluated on the optimum and scaling laws are derived. Finally, based on these results, the topologies with and without back-iron are compared. In the iron-less topologies, the Halbach structure (Top 4) gives better result than the other topologies. Thanks to the use of back-iron, the results on the magnet volume for Top 1, with only axially polarised magnet, are close to results for Halbach iron-less topology (Top 4) but due to manufacturing consideration, Top 1 has to be considered as a competitive topology.

\section{Acknowledgement}

This work was supported by the Walloon Region. 


\section{References}

Bekinal S. I., Anil T. R. R., and Jana S., Analysis of radial magnetized permanent magnet bearing characteristics (2013), Progress In Electromagnetics Research B, vol. 47, pp. 87-10.

Halbach K., Design of permanent multipole magnets with oriented rare earth cobalt material (1980), Nuclear instruments and methods, Vol. 169, pp. 110.

Jungmayr G. , Marth E., Amrhein W., Berroth H.-J., and Jeske F., Analytical stiffness calculation for permanent magnetic bearings with soft magnetic materials (2014), IEEE Transactions on Magnetics, vol. 50, no. 8, pp. 1-8.

Moser R., Sandtner J. and Bleuler H., Optimization of Repulsive Passive Magnetic Bearings (2006), IEEE transactions on magnetics, Vol. 42, No. 8.

Plonsey R. E. C. R., Principes and Applications of Electromagnetic Fields (1961).

Ravaud R., Lemarquand G., and Lemarquand V., Force and stiffness of passive magnetic bearings using permanent magnets. Part 1: Axial magnetization (2009), IEEE Transactions on Magnetics, vol. 45, no. 7, pp. 2996-3002.

Sotelo G. G. , de Andrade Jr R., and Ferreira A. C., Magnetic bearing sets for a flywheel system (2007), IEEE Transactions on Applied Superconductivity, vol. 17, no. 2, pp. 2150-2153.

Van Beneden M., Kluyskens V. and Dehez B., Comparison between optimized geometries of permanent magnet ironless load compensators (2015), INTERNATIONAL SYMPOSIUM on ELECTROMAGNETIC FIELDS (ISEF), Valencia.

Van Beneden M., Kluyskens V. and Dehez B., Optimal sizing and comparison of permanent magnet thrust bearings (2017), IEEE transactions on magnetics, Vol.53, No. 2.

Yonnet J. P., Radial and thrust permanent magnet bearings (2017), Techniques de l'Ingenieur, traite Genie Electrique. (in French)

Yonnet J.-P., Passive magnetic bearings with permanent magnets (1978), IEEE Transactions on Magnetics, vol. 14, no. 5, pp. 803-805.

Yonnet J.-P., Lemarquand G., Hemmerlin S., and Olivier-Rulliere E., Stacked structures of passive magnetic bearings (1991), Journal of Applied Physics, vol. 70, p. 6633

Yoo S.-Y. , Kim W.-Y., Kim S.-J., Lee W.-R., Bae Y.-C., and Noh M., Optimal design of non-contact thrust bearing using permanent magnet rings (2011), International Journal of Precision Engineering and Manufacturing, vol. 12, no. 6, pp. 1009-1014. 